\title{
Correlation of Swirl Number for a Radial-Type Swirl Generator
}

\author{
H. J. Sheen \\ W. J. Chen \\ S. Y. Jeng \\ T. L. Huang \\ Institute of Applied Mechanics, \\ National Taiwan University, \\ Taipei 10764, Taiwan
}

\begin{abstract}
- An experimental investigation was undertaken to derive a new correlation for the swirl number of a radial-type swirl generator under various Reynolds numbers and various vane angle conditions. A radial-type swirl generator with 16 rotary guide vanes was used to generate an annular swirling jet flow. The Reynolds numbers ranged from 60 to 6000 , and the vane angles from $0^{\circ}$ to $56^{\circ}$. Quantitative measurements for the velocities were made by using an optical method of laser-Doppler anemometry (LDA). Three-component velocity profiles of axial, radial, and azimuthal components at the swirling jet exit were measured for various flow conditions. A flow visualization method using smoke-wire and still photography was also applied to observe the flow patterns of the recirculation region behind the circular bluff body. Under low Reynolds number conditions, the swirl strength was found to be strongly dependent on the Reynolds number as well as on the guide vane angle. Based on the experimental results, a modified swirl number $S$ is derived to characterize the swirling flow, which is useful for the design of a swirl generator.
\end{abstract}

Keywords: swirl generator, swirl number, vane angle, laser-Doppler anemometry

\section{INTRODUCTION}

A flow with spiral motion in a tangential direction in addition to axial and radial directions is in general regarded as a swirling flow. The swirling velocity component is imparted to the flow normally via the use of three methods:

1. Axial plus tangential entry of the fluid stream or directly tangential to it

2. The use of turning vanes in an axial tube

3. Rotation of mechanical devices to impart swirling motion to the flow

The use of a swirling flow obtained with a swirl generator improves flame stability in a combustion chamber by forming a toroidal recirculation zone. The flame length can also be reduced, and thus the size of the combustion chamber can be minimized. Furthermore, the introduction of swirling motion to the jet flow can lead to a higher ambient entrainment flow and enhance flow mixing, particularly in the shear layer region. The aerodynamic properties of swirling flow have been studied by a number of investigators (e.g., Syred and Beér [1] and Lilley [2]).

Chigier and Chervinsky [3] used a five-hole spherical impact probe to study an axisymmetric turbulent single swirling jet flow and found that the axial and azimuthal velocity profiles could be approximated by using Gaussian error curves and third-order polynomials. Vu and Gouldin [4] studied a model combustor composed of two confined coaxial swirling jets under noncombusting conditions and found that the recirculation zone was larger for the counterswirl case than for the coswirl case. Syred and Beér [1] concluded that the efficiency of a radial-type guide vane swirler is higher than that of axial-type and axial-plustangential swirlers. Martin [5] reviewed the various designs and performance of axial guide vane swirlers and analyzed their efficiencies, swirl strength, and pressure drop by using axial force thrust and swirl reaction torque. For a swirling flow with a center bluff body, Weber and Dugué [6] concluded that the effects of the bluff body were comparable to the swirl-induced effects when the blockage ratio was 0.7 .

What parameter to use to characterize the swirl strength of a swirling flow is a very important consideration. Some researchers have used the vane angle $\phi$ of the swirler directly (e.g., Yoon and Lilley [7]). Escudier and Keller [8] made detailed flow field measurements on an isothermal annular swirling flow with a bluff body and proposed a parameter $\beta$, obtained by applying potential flow theory for a radial guide vane swirler. The parameter $\beta$ was defined as the ratio of the azimuthal to the axial velocity components at the surface of the central cylinder within the annular cylindrical section and independent of the

Address correspondence to Dr. Horn-Jiunn Sheen, Associate Professor, Institute of Applied Mechanics, National Taiwan University, Taipei 10764, Taiwan, ROC. 
Reynolds number. Martin [5] proposed another parameter of geometric swirl number. The other parameter for combustor applications is the swirl number, which is the ratio of the axial flux of the angular momentum to the axial flux of the axial momentum, and this parameter was more commonly used by most previous researchers to study topics related to swirling flows.

However, the relation between the swirl number $S$ and the vane angle of the swirler $\phi$ under various flow conditions has rarely been studied. Lilley [2] found that the swirl number was proportional to $\tan \phi$. In previous research, the swirl number was expressed as a function of $\phi$ only. The Reynolds number influence was usually assumed to be negligible for the estimation of the swirl number. The present investigation was therefore conducted to study the effects of the Reynolds number and the vane angle of the guide blade on the swirl number for a swirling jet flow from a radial-type swirl generator. The experimental system employed a radial-type swirl generator with 16 guide vanes that could be adjusted to various angles. A nonintrusive optical method with laser-Doppler anemometry (LDA) was used for the measurements of three-component velocity information at the jet flow exit under various flow conditions. The axial and azimuthal momentum fluxes could thus be computed according to the measured velocity distributions. The swirl strength was found to be strongly dependent on the flow Reynolds number and the vane angle for low Reynolds number conditions. A modified correlation for the swirl number of a radial-type swirl generator was derived as a function of both the Reynolds number and the vane angle.

\section{EXPERIMENTAL ARRANGEMENT}

The schematic diagram of the experimental setup is shown in Fig. 1. The air stream from two 11-kW (15-hp) air compressors passed through a dehumidifier filter to a $3.5-\mathrm{m}^{3}$ storage tank. The tank was used to eliminate the unstable stroke effect and to ensure a regulated and stable air supply. An air filter and a pressure regulation valve placed at the outlet of the tank were used to clean and control the air flow. A vortex flow meter for higher flow rate measurements and a rotameter for lower flow rate measurements were also placed at the outlet of the storage tank to monitor the air flow.

The detailed geometry of the radial-type swirl generator is shown in Fig. 2. The air flow passed through a curved settling chamber into a swirl generator with 16 guide vanes. The angle of the guide vanes was controlled by a stepping motor with an angle decoder and could be adjusted from $0^{\circ}$ to $56^{\circ}$ with a resolution of $0.1^{\circ}$. The swirling jet flow was then sent to the annular test section with a concentric cylinder. The annular air flow region had an

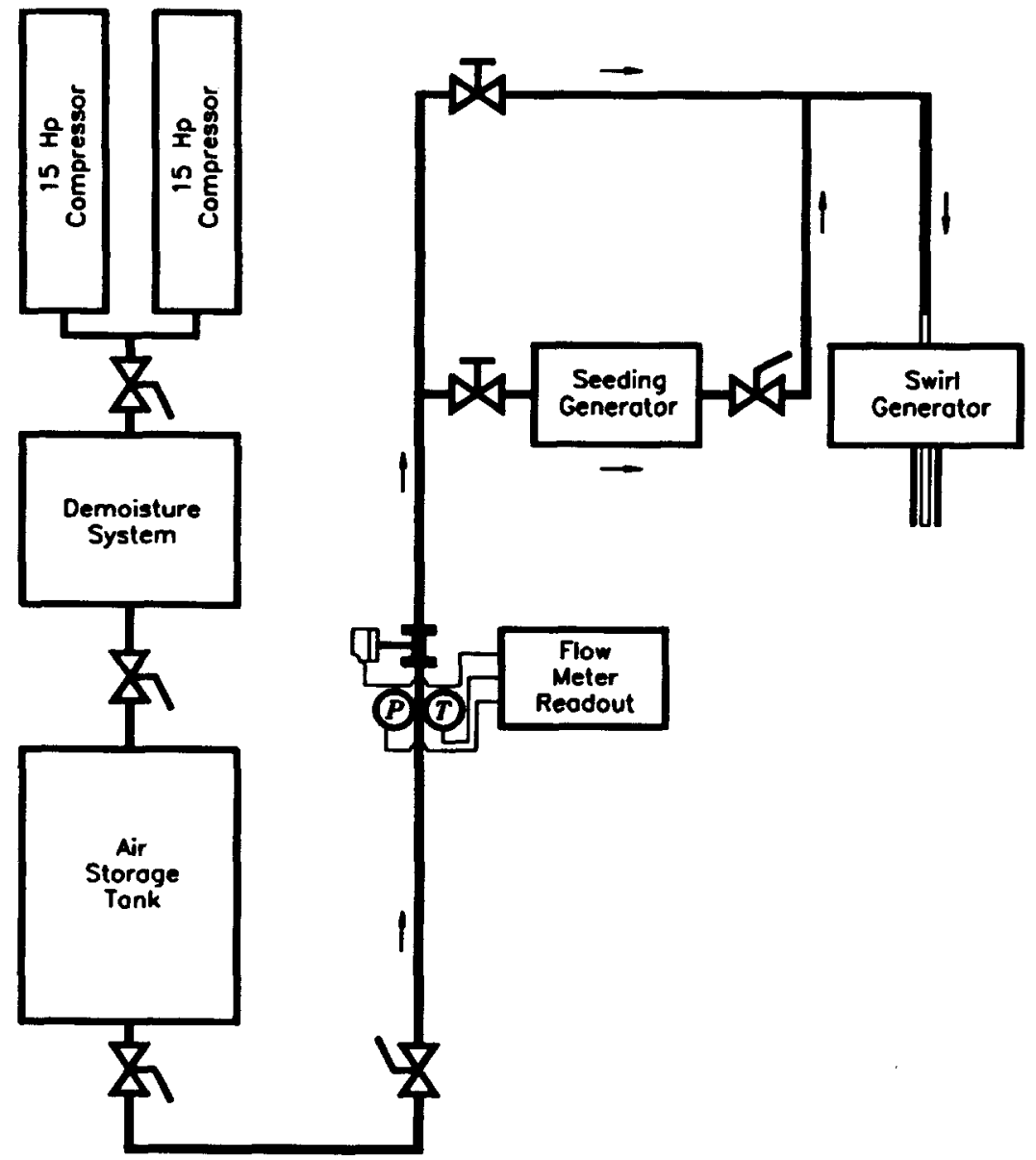

Figure 1. Schematic diagram of the experimental setup. 

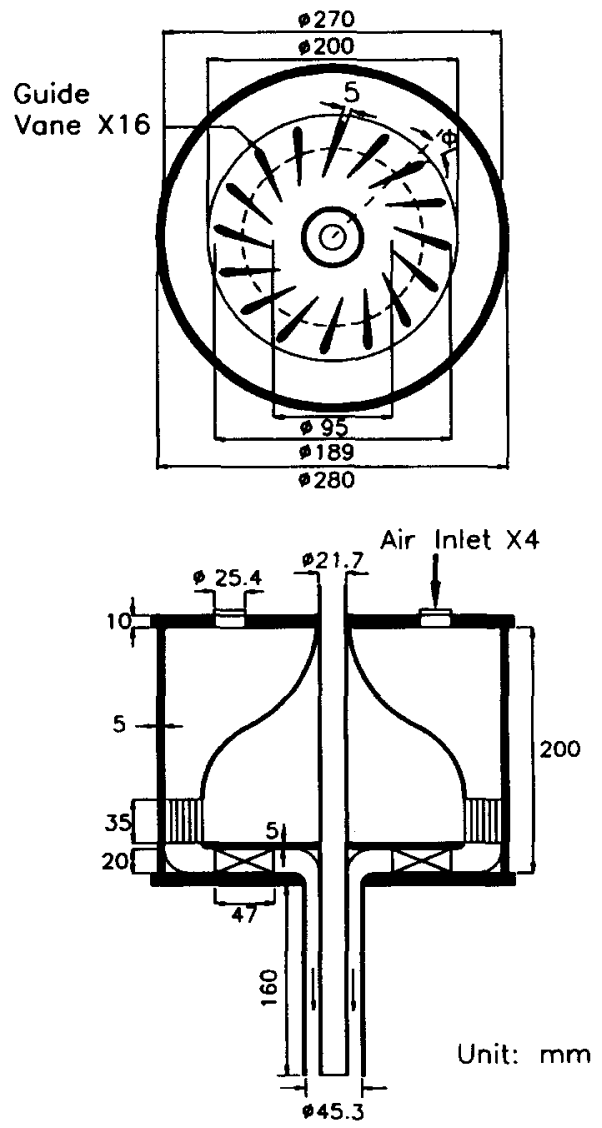

Figure 2. Schematic diagram of the radial-type swirl generator.

inner diameter $d$ of $21.7 \mathrm{~mm}$ and an outer diameter $D$ of $45.3 \mathrm{~mm}$. LDA measurements were performed at the jet flow exit of $x / D=0.09$.

A four-beam, two-component forward-scattering LDA system was placed on a computer-controlled traversing system to perform the velocity measurements for the swirling flow. A 3-W argon ion laser with blue and green beam wavelengths of 488 and $514.5 \mathrm{~nm}$, respectively, was used as the light source. The beam space was $50 \mathrm{~mm}$, and the focusing length was $350 \mathrm{~mm}$. Two Bragg cells for each component were used to extend the measurement range and for the reversed flow measurements. A beam expander, expansion ratio 2.27 , was used to reduce the measuring volume and to increase the signal-to-noise ratio. Two counter-type signal processors with a DMA interface linked to a personal computer were used for data acquisition.

A flow visualization technique using a smoke wire and a still camera was applied for qualitative flow pattern observation of the recirculation zone behind the bluff body. Twisted stainless steel wires with a diameter of $0.1 \mathrm{~mm}$ were placed across the center plane of the jet flow to generate smoke streaks and to make the flow field observable. A $100-\mathrm{W}$ arc lamp was used as the light source for the still camera.

The uncertainty of the air flow rate was estimated to be a maximum of about $1 \%$. The LDA system was mounted on an $X-Y-Z$ traversing system for measurement position adjustment. The precision of the traversing system was $25 \mu \mathrm{m}$ for the $Y$ and $Z$ axes and $2.5 \mu \mathrm{m}$ for the $X$ axis. In this experiment, the uncertainties for LDA velocity measurements were estimated to be $3 \%$ based on the procedure given in [9]. The uncertainty for the measured Reynolds number was $3 \%$, and that for the swirl number was $5 \%$ in the present experiment.

\section{RESULTS AND DISCUSSION}

In this study, the controlled parameters included the vane angle $\phi$ and the Reynolds number, $\operatorname{Re}=U_{\mathrm{m}} d / \nu$, where $U_{\mathrm{m}}$ is the volumetric mean axial velocity, $d$ is the diameter of the circular bluff body, and $\nu$ is the kinematic viscosity of air. The range of Reynolds numbers was 60-6000 in this experiment, and the vane angle ranged from $0^{\circ}$ to $56^{\circ}$. All the experiments were conducted under noncombusting conditions.

\section{Mean Velocity Profiles at the Jet Exit}

Figure 3 shows the LDA measurement results of the mean velocity profiles of the axial, radial, and azimuthal components $U, V$, and $W$ along the radial direction at the jet exit of the annular region, $x / D=0.09$, for various Reynolds numbers and vane angles. The coordinates used included $r$ for the radial direction, $\theta$ for the azimuthal direction, and $x$ for the axial direction. The results show that the swirling jet flow was symmetric at the jet exit of the annular pipe. The flow rate of the swirling jet could be
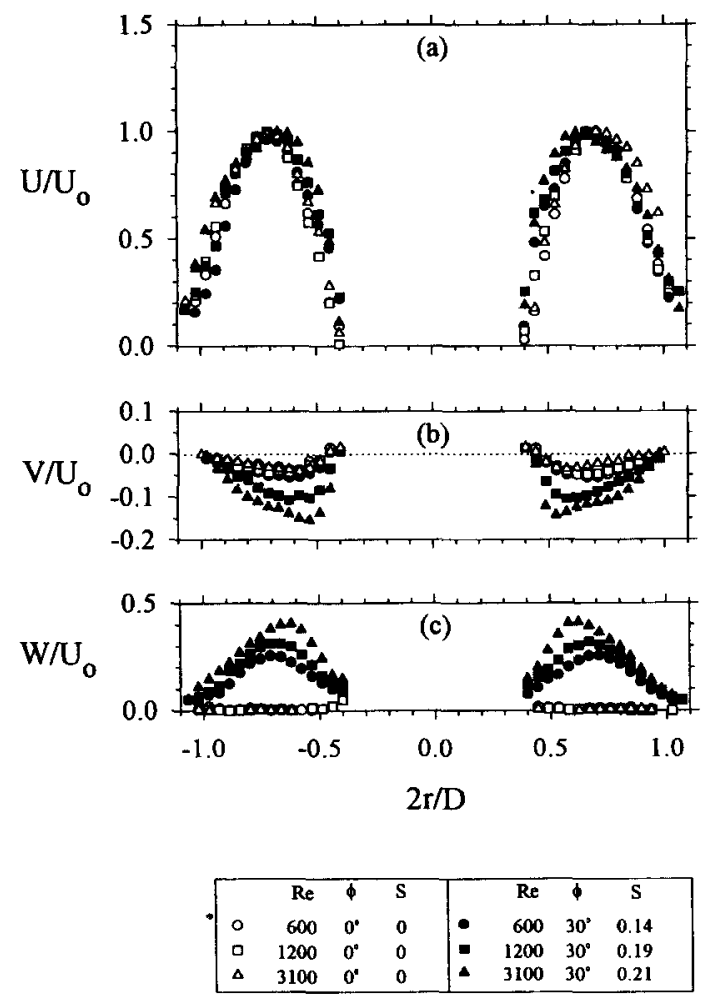

Figure 3. LDA measurements of the mean velocity profiles at the jet exit $(x / D=0.09)$. (a) Axial velocity; (b) radial velocity; (c) azimuthal velocity. 
obtained by using the axial velocities:

$$
Q=\int U d A=\int_{0}^{2 \pi} \int_{0}^{D / 2} U r d r d \theta
$$

The flow rates computed from the measured results in two radial directions were nearly the same, with a difference of less than $3 \%$. Moreover, the normalized axial velocities, $U / U_{0}$, where $U_{0}$ is the maximum axial velocity, of the flow under each Reynolds number and vane angle condition were found to be nearly the same.

The three component velocity profiles at the jet exit of $x / D=0.09$ for the flow with $\operatorname{Re}=600$ and various vane angles are given in Fig. 4. The results of the axial velocity profiles show that the flow was a fully developed laminar flow through an annulus and kept the same profile under various vane angles. The negative values of the radial velocity were increased as the vane angle increased. This result shows that the pressure behind the bluff body became lower when the vane angle was increased. Results of flow visualization, as given in Fig. 5 for vane angles of $0-50^{\circ}$, show that the recirculation zone became smaller as the vane angle was increased.

Figure 6 shows the results of the axial, radial, and azimuthal velocities at the jet exit for the case of $\mathrm{Re}=$ 3100 . The results of the axial velocity profiles reveal that the flow was a fully developed turbulent flow in an annulus. Further, the measured velocity profiles were independent of the vane angle. The velocity profiles with various vane angles were different from those of laminar flow cases such as $\operatorname{Re}=600$. However, the location for the maximum azimuthal velocity moved toward the jet flow central axis as the vane angle increased. The normalized radial and azimuthal velocities, $V / U_{0}$ and $W / U_{0}$, increased when Re increased as seen from Figs. 4 and 6 for $\operatorname{Re}=600$ and 3100 and from other experimental cases.

\section{New Correlation for Swirl Number}

As seen in Fig. 3, the normalized azimuthal velocities were variant for the swirling flow as the Reynolds number was changed although the vane angle was kept the same. Thus the influence of the Reynolds number could not be neglected for the estimation of the flow's swirl strength. The swirl effect on the flow was more pronounced when the Reynolds number was increased. Hence, the proper parameters to characterize the swirl effect for a swirler should include both the vane angle and the Reynolds number.

The degree of swirl for a swirling flow is usually characterized by the swirl number, as was originally proposed by Chigier and Beér [10]:

$$
S_{0}=\frac{G_{\theta}}{R G_{x}},
$$

where $G_{\theta}$ stands for the axial flux of the tangential momentum, $G_{x}$ is the axial flux of the axial momentum, and $R$ is the outer radius of the annulus. These two parameters, $G_{\theta}$ and $G_{x}$, can be expressed as

$$
\begin{gathered}
G_{\theta}=\int_{0}^{R}(W r) \rho U 2 \pi r d r=k_{1}, \\
G_{x}=\int_{0}^{R} 2 \pi r \rho U^{2} d r+\int_{0}^{R} 2 \pi r p d r=k_{2},
\end{gathered}
$$

where $U, W$, and $p$ are the axial and azimuthal components of the velocity and static pressure, respectively, and $k_{1}$ and $k_{2}$ are constants for an unconfined swirling jet flow. For a confined swirling flow, $G_{\theta}$ and $G_{x}$ will not remain constant due to the effects of the confined boundary.

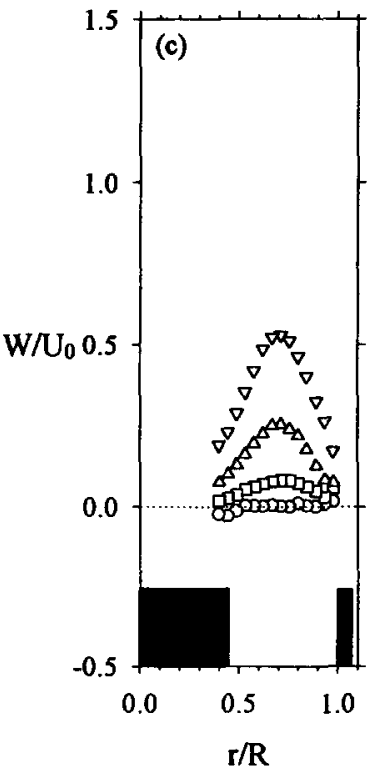

Figure 4. LDA measurements of the mean velocity profiles at the jet exit $(x / D=0.09)$ for various vane angles at $\operatorname{Re}=600$. (a) Axial velocity; (b) radial velocity; (c) azimuthal velocity. 


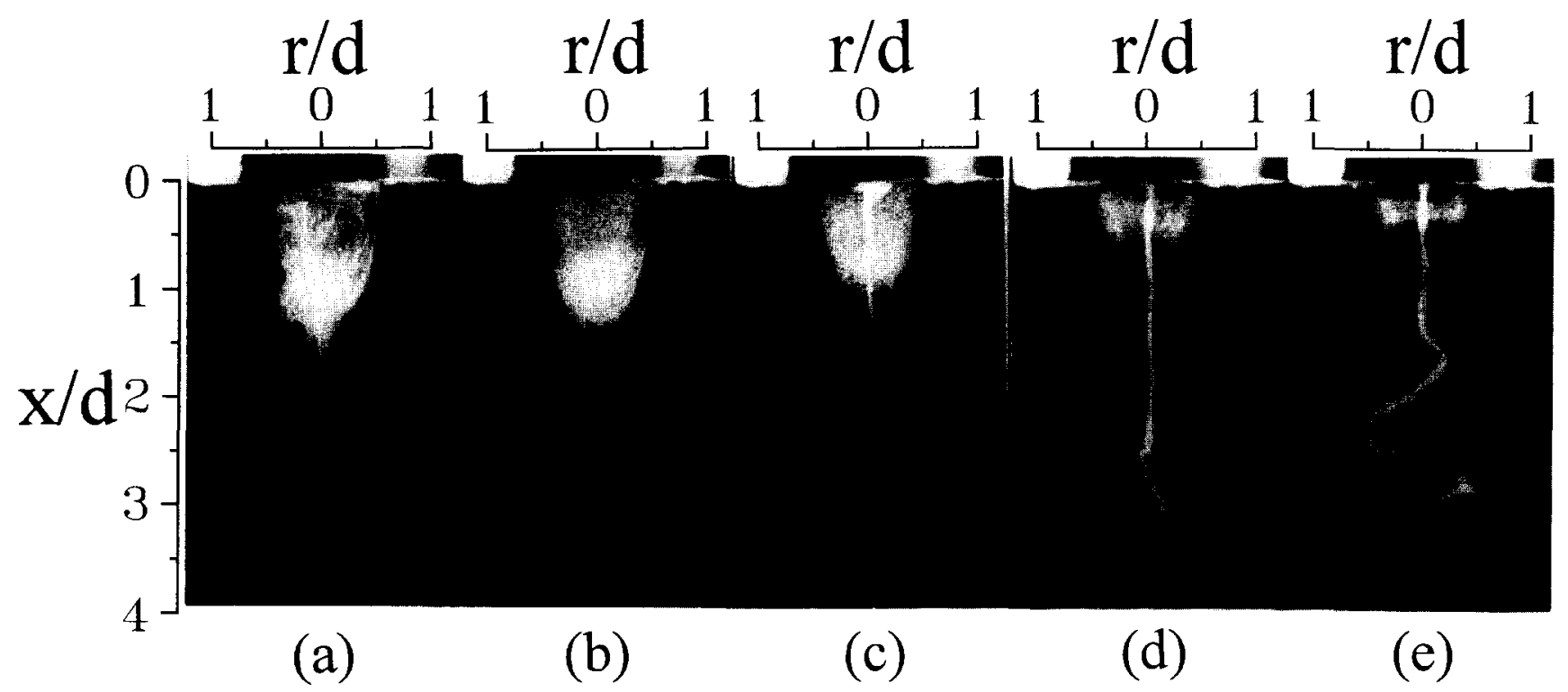

Figure 5. Flow patterns behind the bluff body observed by using the smoke-wire flow visualization technique at $\mathrm{Re}=600$. (a) Nonswirling flow, $\phi=0^{\circ}$; (b) swirling flow, $\phi=20^{\circ}$; $(c)$ swirling flow, $\phi=30^{\circ}$; (d) swirling flow, $\phi=40^{\circ}$; (e) swirling flow, $\phi=50^{\circ}$.

The static pressure can be approximated from the measured azimuthal velocities; then Eq. (4) can be simplified to

$$
G_{x}=2 \pi \int_{0}^{R} \rho r\left(U^{2}-\frac{1}{2} W^{2}\right) d r
$$

Thus, another swirl number is deduced as

$$
S_{0}^{\prime}=\frac{\int_{0}^{R} r^{2} U W d r}{R \int_{0}^{R} r\left[U^{2}-(1 / 2) W^{2}\right] d r},
$$

which has also been used by many investigators (e.g., Ribeiro and Whitelaw [11]). Chigier and Beér [10] also calculated the radial pressure distribution from the radial distribution of azimuthal velocity by integrating the equation

$$
\frac{d p}{d r}=\rho \frac{W^{2}}{r}
$$

For a high degree of swirl, good agreement was found between the calculated and measured values. However, for a low degree of swirl, the agreement was not as good because the effects of friction may not be negligible.
Figure 6. LDA measurements of the mean velocity profiles at the jet exit $(x / D=0.09)$ for various vane angles at $\operatorname{Re}=3100$. (a) Axial velocity; (b) radial velocity; $(c)$ azimuthal velocity.
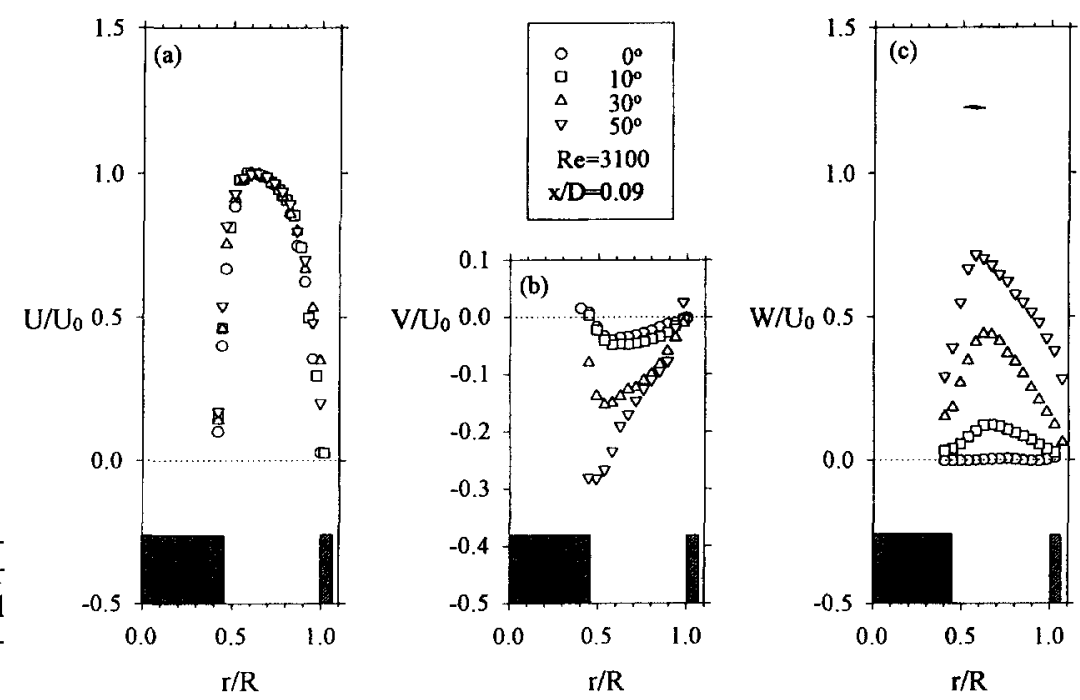
It is very difficult to directly estimate the pressure integral term because the static pressures are strongly dependent on the geometry of the swirler. According to Beér and Chigier [12], Martin [5], and Weber and Dugué [6], the axial flux of axial momentum, $G_{x}$, can be obtained with good approximation by eliminating the pressure term of Eq. (4). Thus, the modified swirl number $S$ defined in Eq. (2), can generally be expressed as

$$
S=\frac{G_{\theta}}{2 \pi R \int_{0}^{R} \rho U^{2} r d r}=\frac{\int_{0}^{R} U W r^{2} d r}{R \int_{0}^{R} U^{2} r d r} .
$$

This modified swirl number $S$ has a greater physical meaning than the two parameters $S_{0}$ and $S_{0}^{\prime}$, because $S$ is linearly proportional to the ratio of volumetric mean azimuthal to axial velocity, $S=0.75 W_{\mathrm{m}} / U_{\mathrm{m}}$, for various Reynolds numbers as shown in Fig. 7 , which shows the measured results.

For a swirling flow from a radial-type swirler with guide vane cascades, the angular momentum flux can be expressed as [12]

$$
G_{\theta}=\sigma \frac{\dot{M}^{2}}{2 \pi \rho B},
$$

where $\dot{M}$ is the mass flow rate, $B$ is the axial width of the annular channel, and $\sigma$ is the ratio of the mean tangential and radial velocity components at the swirler exit, which has a radius $R_{1}$ as shown in Fig. 8. Since $\sigma$ is dependent only on the geometrical dimensions of the guide vane and the vane angle $\phi$, the angle of the guide vane with respect to the radial direction, the value of $\sigma$ can be obtained as

$$
\sigma(\phi)=\frac{1}{1-\psi}\left(\frac{\tan \phi}{1+\tan \phi \tan (\pi / z)}\right),
$$

where $\psi=z s / 2 \pi R_{1} \cos \phi$ is a blockage factor that comes from the finite thickness of the guide vanes, $z$ is the number of guide vanes, and $s$ is the thickness of the guide vanes.

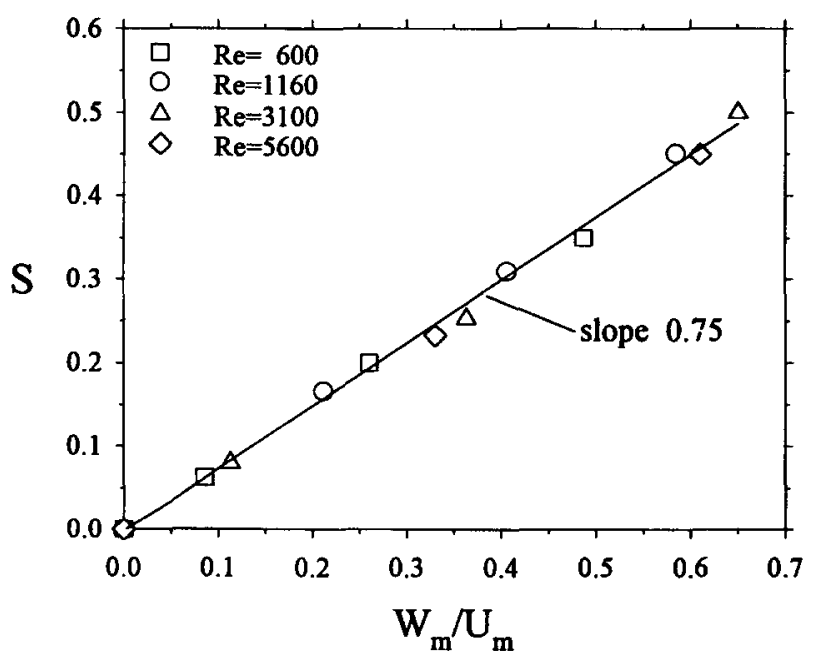

Figure 7. Correlation of swirl number $S$ with the ratio of the volumetric mean azimuthal to axial velocity, $W_{\mathrm{m}} / U_{\mathrm{m}}$.

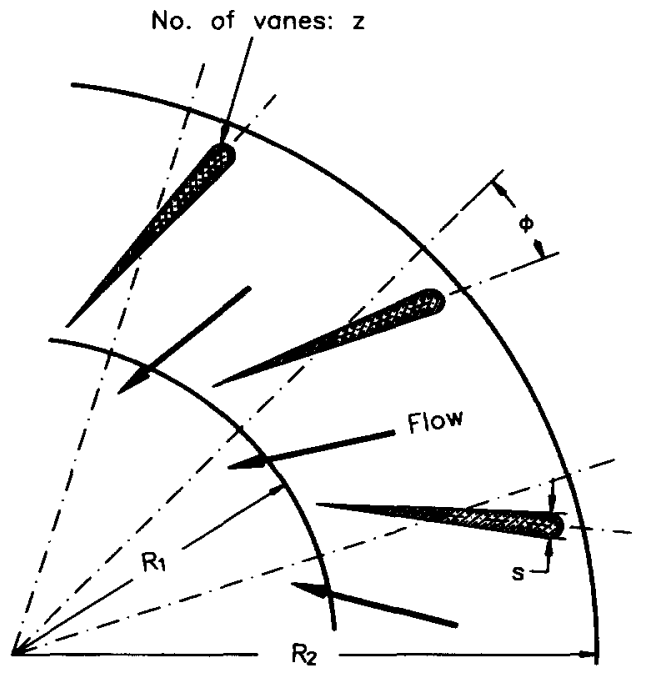

Figure 8. Diagram of the guide vane cascade in the swirl generator.

Substituting Eqs. (9) and (10) into Eq. (8), we obtain

$$
S=\frac{\dot{M}^{2}}{4 \pi^{2} \rho B R \int_{0}^{R} \rho U^{2} r d r} \sigma(\phi) .
$$

Since the velocity profiles of mean axial velocity along the radial direction are almost the same as for flows with various vane angles as shown in Figs. 4 and 6, Eq. (11) can be further reduced to

$$
S=C_{1}(\operatorname{Re}) \sigma(\phi),
$$

where $C_{1}$ is a function of Re with the guide vane at a fixed angle. Experimental verification of Eq. (12) is demonstrated in Fig. 9 for the relationship between $\phi$ and $S$ for

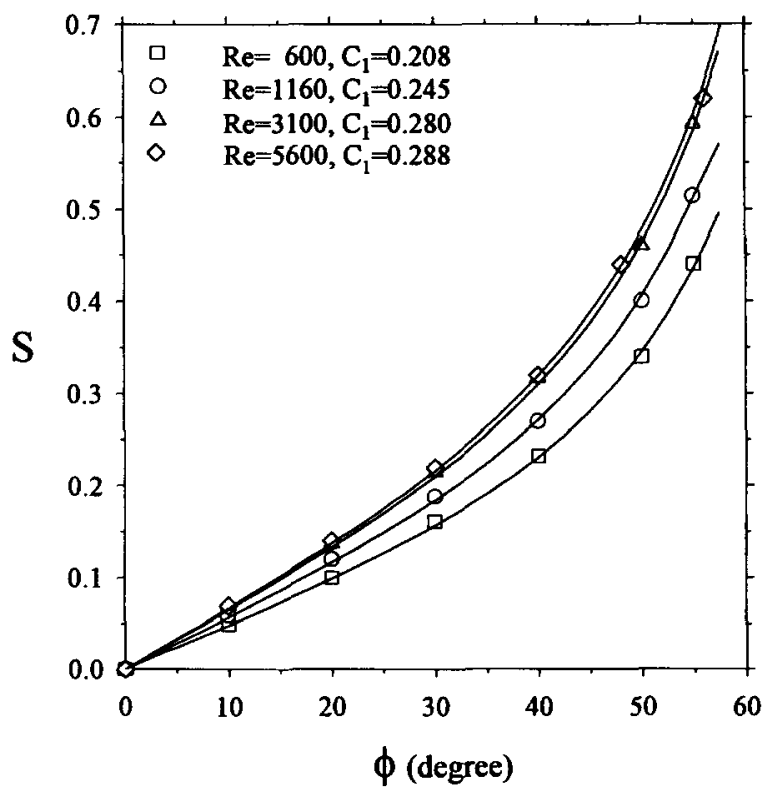

Figure 9. Correlations of the swirl number $S$ with the vane angle $\phi$ for the cases of $\operatorname{Re}=600,1160,3100$, and 5600 . 
the cases of $\mathrm{Re}=600,1160,3100$, and 5600. In Fig. 9, the value of $S$ for each vane angle case was computed by using Eq. (8) according to the measured axial and azimuthal velocity profiles. With Eq. (10), the values of the constant $C_{1}$ could thus be obtained by using curve fitting and was found to be $0.208,0.245,0.280$, and 0.288 for flows with $\operatorname{Re}=600,1160,3100$, and 5600 , respectively. Similarly, the values of $C_{1}$ for other Reynolds number cases could also be calculated, and the results are given in Fig. 10. Finally, a general correlation of $C_{1}$ for various Reynolds number conditions could thus be obtained as

$$
C_{1}(\operatorname{Re})=0.28 \times\left[1-\operatorname{sech}\left(0.026 \operatorname{Re}^{0.67}\right)\right] .
$$

From Eqs. (10), (12), and (13), the swirl number $S$ under various Reynolds numbers and guide vane angles $\phi$ for a radial-type swirl generator can be derived. Further, from Eq. (13) and the velocity characteristics for the swirling flow in the laminar, transitional, and turbulent flow regimes, the values of $C_{1}$ are increased linearly with $\mathrm{Re}$ in the laminar flow regime, then increased nonlinearly with $\mathrm{Re}$ in the transitional flow regime, and finally approach a constant in the turbulent flow regime.

Regarding the design of a swirl generator, when $\operatorname{Re}<$ 3000 , the swirl number increases as the Reynolds number increases under the same vane angle condition, as shown in Fig. 10. For $\mathrm{Re}>3000$, the values of $C_{1}$ approach a constant, and the swirl number is dependent only on the vane angle of the swirl generator and is independent of the Reynolds number. Thus, for the design of a radial-type swirl generator, one should take the Reynolds number or the flow rate into account to evaluate the swirl number in addition to other considerations such as efficiency and vane geometry.

\section{PRACTICAL SIGNIFICANCE}

We found that the swirl number of a radial-type swirl generator was dependent not only on the guide vane angle but also on the Reynolds number. The Reynolds number or the flow rate was found to have a great influence on the swirl number when the flow was in the lower $\mathrm{Re}$ regime. We also derived a modified correlation for the swirl number that correlated with the guide vane angle, the number of guide vanes, the Reynolds number, and other parameters. The results could be applied to the design of a swirl generator for a combustion chamber and used in other applications.

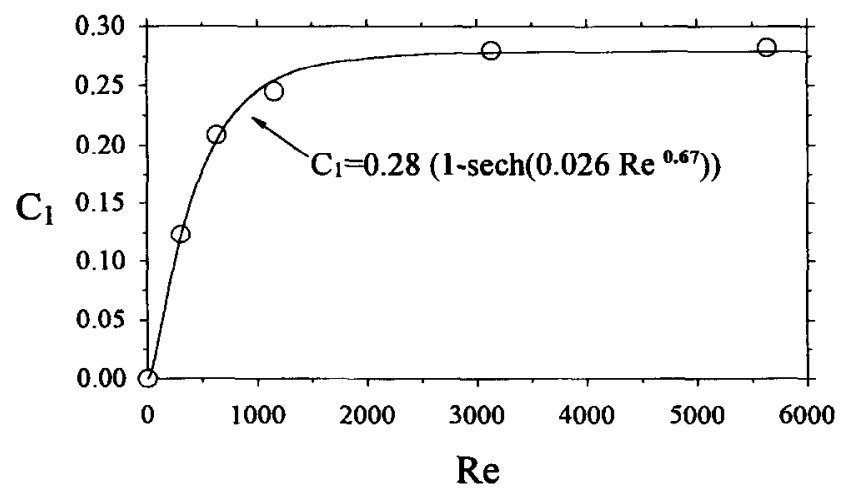

Figure 10. Correlation for the constant $C_{1}$ of the swirl number $S$ with various Reynolds numbers.

\section{CONCLUSIONS}

An experimental investigation was carried out to study the effects of the flow's Reynolds number and the guide vane angle on the flow structure at the jet exit of a radial-type swirl generator. A modified swirl number for the swirl generator was derived that was found to be strongly dependent on the Re in low Reynolds number cases. Following is a summary of the important findings in this experimental investigation.

1. The degree of swirl of a swirling flow was found to be dependent upon the flow's Reynolds number and the vane angle for a radial-type swirl generator. The modified swirl number $S$ correlated with both the Reynolds number and the vane angle, and the correlation derived was $S=C_{1}(\operatorname{Re}) \sigma(\phi)$.

2. In this experiment with $\mathrm{Re}=300-6000$, the swirl number $S$ was found to be linearly proportional to the ratio of volumetric mean azimuthal to axial velocity, and the relation was $S=0.75 W_{\mathrm{m}} / U_{\mathrm{m}}$.

3. For a lower Reynolds number, such as $\mathrm{Re}=600$, the radial distributions of the mean axial and azimuthal velocities were similar for various vane angles. They could be approximated by parabolic curves. For a higher Reynolds number, such as $\mathrm{Re}=3100$, the peak value of azimuthal velocity moved toward the jet flow central axis when the swirl angle was increased.

4. At $x / D=0.09$, which is near the jet exit, the flow had a radial velocity with a direction toward the jet center. The magnitude of the radial velocity increased and the recirculation zone became smaller as the swirl number increased.

This research was supported by the National Science Council of Taiwan, Republic of China, under grant number NSC83-0401-E002134. We are also grateful to Potters-Ballotini GmbH of Germany for their very generous supply of glass beads.

\section{NOMENCLATURE}

$B$ width of guide vane, $20 \mathrm{~mm}$

$d$ diameter of cylindrical bluff body, $21.7 \mathrm{~mm}$

$D$ outer diameter of annulus, $45.3 \mathrm{~mm}$

$G_{x}$ axial flux of axial momentum, $\mathrm{kg} \mathrm{m} / \mathrm{s}^{2}$

$G_{\theta}$ axial flux of angular momentum, $\mathrm{kg} \mathrm{m}^{2} / \mathrm{s}^{2}$

$\dot{M}$ mass flow rate, $\mathrm{kg} / \mathrm{s}$

$p$ static pressure, $\mathrm{N} / \mathrm{m}^{2}$

$R$ outer radius of annulus $(=D / 2), 22.65 \mathrm{~mm}$

Re Reynolds number $\left(=U_{\mathrm{m}} d / \nu\right)$, dimensionless

$R_{1} \quad$ inner radius of guide vane cascade, $47.5 \mathrm{~mm}$

$R_{2}$ outer radius of guide vane cascade, $94.5 \mathrm{~mm}$

$s$ thickness of guide vane, $\mathrm{mm}$

$S$ modified swirl number, dimensionless

$S_{0} \quad$ swirl number $\left(=G_{\theta} / G_{x} R\right)$, dimensionless

$S_{0}^{\prime} \quad$ swirl number $\left[=\int_{0}^{R} r^{2} U W d r /\left(R \int_{0}^{R} r\left(U^{2}-\right.\right.\right.$ $\left.\left.\left.W^{2} / 2\right) d r\right)\right]$, dimensionless

$Q$ volumetric flow rate, $\mathrm{m}^{3} / \mathrm{s}$

$U$ time-mean axial velocity, $\mathrm{m} / \mathrm{s}$

$U_{\mathrm{m}}$ volumetric mean axial velocity, $\mathrm{m} / \mathrm{s}$ 
$U_{0}$ maximum axial velocity, $\mathrm{m} / \mathrm{s}$

$V$ time-mean radial velocity, $\mathrm{m} / \mathrm{s}$

$W$ time-mean azimuthal velocity, $\mathrm{m} / \mathrm{s}$

$W_{\mathrm{m}}$ volumetric mean azimuthal velocity, $\mathrm{m} / \mathrm{s}$

$z$ number of guide vanes in cascade, dimensionless

\section{Greek Symbols}

$\nu$ kinematic viscosity of air, $\mathrm{m}^{2} / \mathrm{s}$

$\phi$ vane angle, deg

$\psi$ blockage factor $[=z s /(2 \pi \cos \phi)]$, dimensionless

\section{REFERENCES}

1. Syred, N., and Beér, J.M., Combustion in Swirling Flows: A Review, Combust. Flame 23, 143-201, 1974.

2. Lilley, D. G., Swirl Flows in Combustion: A Review, $A L A A J$. 15(8), 1063-1078, 1977.

3. Chigier, N. A., and Chervinsky, A., Experimental Investigation of Swirling Vortex Motion in Jets, J. Appl. Mech. 443-451, June 1967.

4. Vu, B. T., and Gouldin, F. C., Flow Measurements in a Model Swirl Combustor, AIAA J. 20(5), 642-651, 1982.

5. Martin, C. A., Aspects of the Design of Swirlers as Used in Fuel
Injectors for Gas Turbine Combustors, ASME Paper No. 87-GT139, 1987.

6. Weber, R., and Dugué, J., Combustion Accelerated Swirling Flows in High Confinements, Prog. Energy Combust. Sci. 18, 349-367, 1992.

7. Yoon, H. K., and Lilley, D. G., Five-Hole Pitot Probe Time-Mean Velocity Measurements in Confined Swirling Flows, AIAA 21st Aerospace Sci. Meeting, Reno, NV, AIAA-83-0315, 1983.

8. Escudier, M. P., and Keller J. J., Recirculation in Swirling Flow: A Manifestation of Vortex Breakdown, ALAA J. 23(1), 111-116, 1985.

9. Moffat, R. J., Describing the Uncertainties in Experimental Results, Exp. Thermal Fluid Sci. 1, 3-17, 1988.

10. Chigier, N. A., and Beér, J. M., Velocity and Static-Pressure Distributions in Swirling Air Jets Issuing from Annular and Divergent Nozzles, J. Basic Eng. 788-796, December 1964.

11. Ribeiro, M. M., and Whitelaw, J. H., Coaxial Jets With and Without Swirl, J. Fluid Mech. 96, 769-795, 1980.

12. Beér, J. M., and Chigier, N. A., Combustion Aerodynamics, Wiley, Inc., New York, 1972.

Received September 8, 1994; revised November 10, 1995 